\title{
Implementation, calibration and operation of a Water Cherenkov Detector at Escuela Politécnica Nacional
}

\author{
Stephany Vargas, ${ }^{a}$ Nicolás Vásquez ${ }^{* a}{ }^{a}$ Scar Martínez ${ }^{b}$ and Andrés Delgado ${ }^{a}$ for \\ the LAGO Collaboration ${ }^{c}$ \\ ${ }^{a}$ Departamento de Física, Escuela Politécnica Nacional \\ Ladrón de Guevara E1-253, Quito, Ecuador \\ ${ }^{b}$ Facultad de Ciencias Físico Matemáticas, Benemérita Universidad Autónoma de Puebla \\ Avenida San Claudio y 18 Sur, Colonia San Manuel, Edificio FM1-101B, Ciudad Universitaria, \\ C.P. 72570, Puebla, México \\ ${ }^{c}$ lagoproject.org, see the full list of members and institutions at \\ lagoproject.org/collab.html \\ E-mail: yostephy@hotmail.com, nicolas.vasquez@epn.edu.ec, \\ omartinefcfm.buap.mx, omartinefcfm.buap.mx
}

This paper describes the installation, calibration and operation of a Water Cherenkov Detector (WCD) at Escuela Politécnica Nacional in Quito-Ecuador at 2810 m.a.s.l. and latitude $0^{\circ} 13^{\prime} 47^{\prime \prime}$ $\mathrm{S}$. The project was developed as part of the Latin American Giant Observatory (LAGO) Collaboration. The instrumentation consists of a polyethylene tank, a 5" PMT (EMI 9530A) and the electronic acquisition system from the LAGO-México project. Data analysis estimates the average time of a pulse to be $154.83 \pm 22.1 \mathrm{~ns}$ for an input voltage set at $1550 \pm 20 \mathrm{~V}$ and a threshold of $22 \mathrm{mV}$. In addition, there are two baseline tendencies, one at $-49.06 \mathrm{mV}$ and another one at $-41.16 \mathrm{mV}$. Moreover, the value of the deposited charge associated to a vertical relativistic muon passing through the detector, the so-called Vertical Equivalent Muon (VEM), was determined from (1) the experiment and from (2) the use of an in-house code developed by the LAGO Collaboration, called 'LagoSim'. The VEM value obtained (1) from the experiment is $3350 \pm 1160 \mathrm{pC}$ and (2) from the simulation is $3800 \pm 250 \mathrm{pC}$. Therefore, the PMT's ability to register the charge is $88 \%$ of the expected value, which is interpreted as a consequence of the life-time quality of the PMT. Finally, muon angular incidence and interaction with materials such as concrete, iron and water are studied to explain the deficit in the charge histogram around $1000 \mathrm{pC}$.

35th International Cosmic Ray Conference - ICRC2017

10-20 July, 2017

Bexco, Busan, Korea

*Speaker. 


\section{Introduction}

In this paper we describe the indirect detection of the muonic component of EAS in a ground based experiment. The experiment is carried out as part of the Latin American Giant Observatory (LAGO) that uses Water Cherenkov Detectors (WCDs) located at some hundred and thousand meters above sea level (m.a.s.1.) [1,2]. The installation and calibration of WCDs had been successfully done in different LAGO sites and are shown in $[3,4,5,6]$.

The LAGO collaboration is unique because it spans between the meridians 60 to 80 perpendicular to the equatorial line and extends towards the southern part of the globe [7]. One of the advantages of this setting is the capability to register in great detail phenomena such as Forbush decreases produced by Coronal Mass Ejections (CMEs) in places with different magnetic rigidity cut off [1]. This is possible because it is known that the flux of cosmic rays depends on the geomagnetic properties asociated with the site and the energy of the primary. In [8] is shown the simulated flux of secondary particles for the city of Quito with and without considering the magnetic rigidity cut off. In the simulation it is emphasized that the maximum flux corresponds to muons, electrons and photons.

The LAGO project in Ecuador began in 2012 with the installation of a WCD (Chimbito detector) in the province of Chimborazo at the University "Escuela Politécnica del Chimborazo (ESPOCH)" [9]. In 2014, a second detector (Panchito detector) was placed at the "Universidad San Francisco de Quito (USFQ)" in the province of Pichincha. Both detectors work with the electronic adquisition system from the Pierre Auger Experiment [8, 10].

The "Escuela Politécnica Nacional" in the city of Quito, Pichincha province, began working in the LAGO collaboration since 2015. A WCD (Politanque detector) was installed, calibrated and operated from June 2015 to February 2016 with the Mexican electronic adquisition system [3]. For the Politanque and Panchito detectors there was a preliminary study published in $[8,11]$.

\section{Methodology}

\subsection{Site}

A WCD named "Politanque" was installed at Escuela Politécnica Nacional at 2800 m.a.s.l. with a mean temperature of $13.9^{\circ}{ }^{1}$. Given that the detector is located below 4000 m.a.s.l. its main purpose is for space weather monitoring [12].

\subsection{Experimental Setup}

Main body and PMT: The largest component of the detector is a commercial black polyethylene tank of $0.81 \mathrm{~m}$ high, $0.24 \mathrm{~m}$ radius and an average volume of $0.15 \mathrm{~m}^{3}$. The sensing part consists of an $130 \mathrm{~mm}$ EMI 9530A photomultiplier tube (PMT). The PMT factory specifications can be found in [13]. The PMT is mechanically fixed to the lid of the tank such that the cathode faces the bottom of the tank. The tank is filled with water up to $70 \mathrm{~cm}$ height and $50 \mathrm{ml}$ of hydrogen peroxide at $90 \%$ purity is added. A black plastic covers completely the detector: sides, top and bottom of the tank; and a thick dark blanket is added.

\footnotetext{
${ }^{1}$ Taken from es.climate-data.org/location/1012
} 
Electronic acquisition system: The PMT is fed with a high voltage source (input voltage of $1550 \mathrm{~V}$, Figure 1). The output signal is connected to a data acquisition board (digitizer and FPGA) designed as part of the EAS-BUAP project. The system is described as: "ADC Dual AD9216-100Ms/s 10-bit resolution with a dynamic conversion range of -1 to 1 volts and 320MHz bandwidth", [3] pg. 36.

Average duration of a pulse: It is found that the average value of a pulse duration is $154.83 \pm 22.1 \mathrm{~ns}$.

\subsection{PMT stability region}

The operation of the PMT depends on the supply voltage and the threshold. Theoretically the photon count in the PMT is non-linear, but in practice it has to be linear in a certain dynamic range [14]. To find this region of work we proceed to take several readings with the oscilloscope of the number of events that occur in $1 \mathrm{~s}$ for different threshold values ranging from $10 \mathrm{mV}$ to $100 \mathrm{mV}$, varying the voltage from $1000 \mathrm{~V}$ to $1700 \mathrm{~V}$.

The objective is to find the region of linearity for the PMT such that a dimensionless count equals $2 \mathrm{mV}$ [3]. In this region, where the slope is a minimum, the range of variance of PMT, amplifier gain, and threshold have the least influence with respect to detection stability [14]. It is found (Figure 1) that for a threshold of $10 \mathrm{mV}$ the linear range is around $1500 \pm 20 \mathrm{~V}$ and for a threshold of $20 \mathrm{mV}$ the linear range is around $1550 \pm 20 \mathrm{~V}$. For higher thresholds the linear behaviour can not be distinguished for the work voltage, so they are discarded. Performing a pre-analysis discarded the threshold of $10 \mathrm{mV}$ as it tends to saturate quickly. Consequently, data is taken with a threshold of $20 \pm 5 \mathrm{mV}$ with a supply voltage of $1550 \pm 20 \mathrm{~V}$.

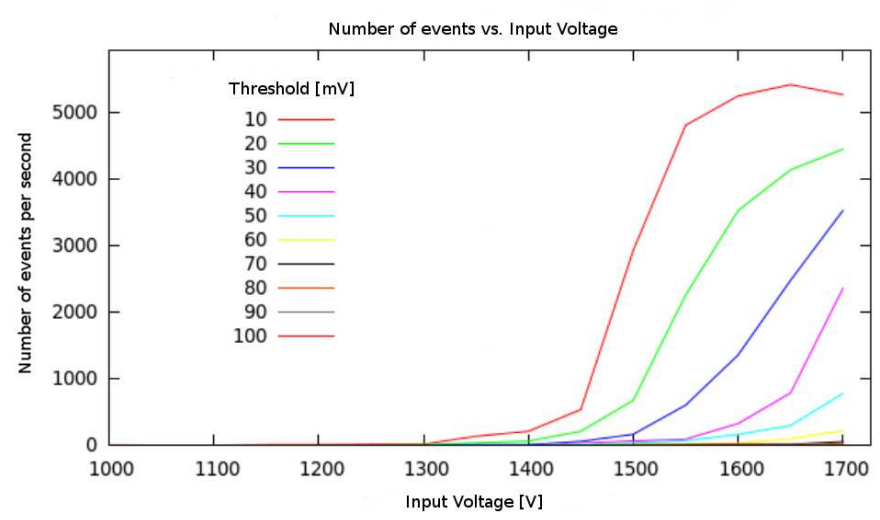

Figure 1: Number of events occurring in $1 \mathrm{~s}$ for different values of threshold $(\mathrm{mV})$ and of input voltage $(\mathrm{V})$.

\section{Results and discussion}

\subsection{Offset}

The data is sorted into 10-minute data files that have its own baseline value. The histogram shows that there are two tendencies for the global baseline, one at $-0.049 \pm 1.873 \times 10^{-5}$ and another at $-0.041 \pm 0,0006$ (Figure 2). This tendency could be due to fluctuations of the main power supply. 


\subsection{Secondary particle detected rate}

The RATE is the number of particles per unit area and unit time. The rate in Quito has been simulated in [8], as a consequence we know that most of the detected particles that will contribute to the rate are electrons/positrons, photons and muons. Muons are the most energetic and have a maximum flow (greater than $10^{4}$ ) between 1 and $10 \mathrm{GeV}$, the flow is reduced exponentially between 10 and $100 \mathrm{GeV}$ with a flow of $\sim 10^{3}$.

The detected particles per second is $4.91 \pm 0.45$ (Figure 5), for an effective area of $0.76 \mathrm{~m}^{2}$, such that it has a rate of $6.406 \pm 0.45 \mathrm{~s}^{-1} \mathrm{~m}^{-2}$. In [8], the flux of particles having an energy around $100 \mathrm{GeV}$ is $600 \mathrm{~s}^{-1} \mathrm{~m}^{-2}$. In [15], for a latitude of $2800 \mathrm{~m} . \mathrm{a}, \mathrm{s}, 1$. , there are approximately 650 $\mathrm{s}^{-1} \mathrm{~m}^{-2}$, such that the detection efficiency is $0.98 \%$ with respect to [15] and of $1.067 \%$ with respect to the simulations carried out by [8].
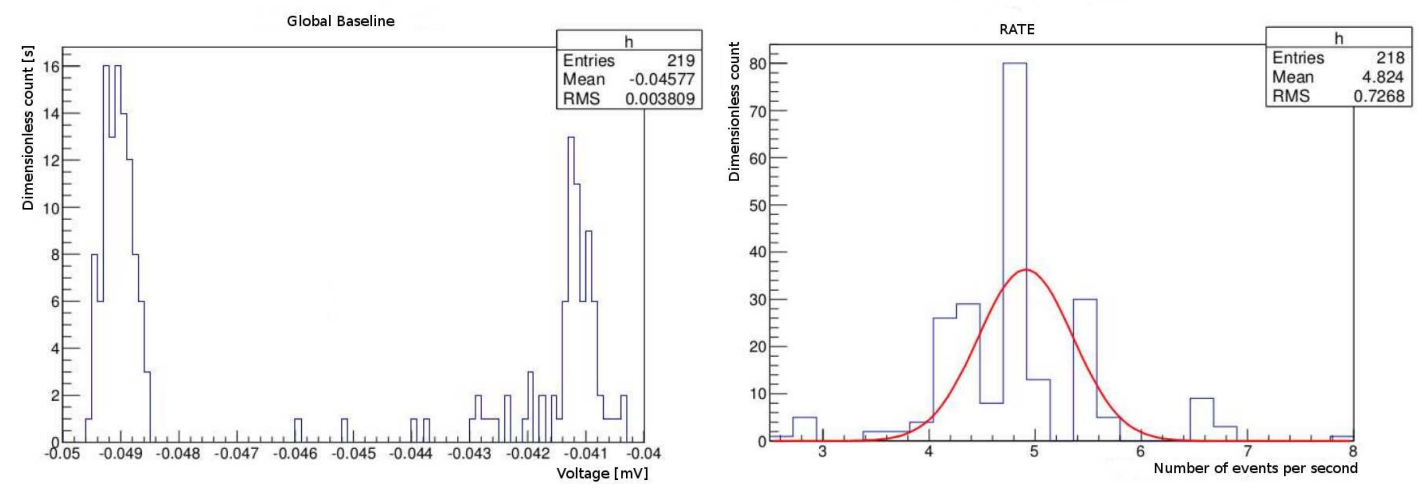

Figure 2: Left: Histogram of the global baseline for 10-minute data files. The baseline shows a duality behaviour with two peaks, one at $-0.049 \pm 1.873 \times 10^{-5}$ and another at $-0.041 \pm 0,0006$. Right: Histogram of the RATE and gaussian fit for 218 entries of 10-minute data files. The curve is fitted with a peak at $4.9 \pm 0.45 \mathrm{~s}^{-1}$ for an effective area of $0.76 \mathrm{~m}^{2}$, such that it has a rate of $6.406 \pm 0.45 \mathrm{~s}^{-1} \mathrm{~m}^{-2}$

\subsection{Charge Distribution Histogram}

\subsubsection{Experiment}

Particles that pass through the detector deposit energy that is measured as a voltage pulse. Considering that a point of a pulse is taken every $10 \mathrm{~ns}$, the charge deposited by a particle is

$$
Q=\int I(t) d t=\frac{1}{R} \int V(t) d t \sim \frac{1}{R} \sum_{0}^{N} V \times \Delta t=\frac{1}{R} \sum_{0}^{N} 10 * V p C
$$

where the voltage $V$ is measured in $\mathrm{mV}$, time is in ns, the resistance $R$ is $50 \Omega$ and the charge $Q$ is in $\mathrm{pC}$. The histogram of the charge of the pulses is denominated a Charge Distribution Histogram (CDH) (Figure 3).

\subsubsection{Simulation LAGOSim}

A complete set of simulations corresponding to the particles that arrive to the detector were made in the framework of the program LAGOSim [6]. LAGOSim is a simplified simulation program developed for the LAGO project detectors. For the simulation presented in this work, the main input parameters are: 
- Altitude: 2800 m.a.s.l.

- Number and position of the PMT's: 1 PMT centered in the upper part of the detector.

- Volume: $0.13 \mathrm{~m}^{3}$.

- PMT area: $\pi * r^{2}=\pi * 52$ inch $^{2}=19.64 \mathrm{inch}^{2}=126.68 \mathrm{~cm}^{2}$.

In each simulation the relative particle flow is: $30 \%$ muons, $23 \%$ electrons/positrons and $47 \%$ gammas. Under these circumstances the obtained CDH is shown in Figure 3. When comparing both figures, we observe that there is a missing region around $800 \mathrm{pC}$ for the observed $\mathrm{CDH}$. The detector is located inside a concrete building ( $4^{\text {th }}$ floor out of 6 floors) in a room with a window. The concrete (and some iron) will act as a shield, while the window will let the particles pass to the detector.
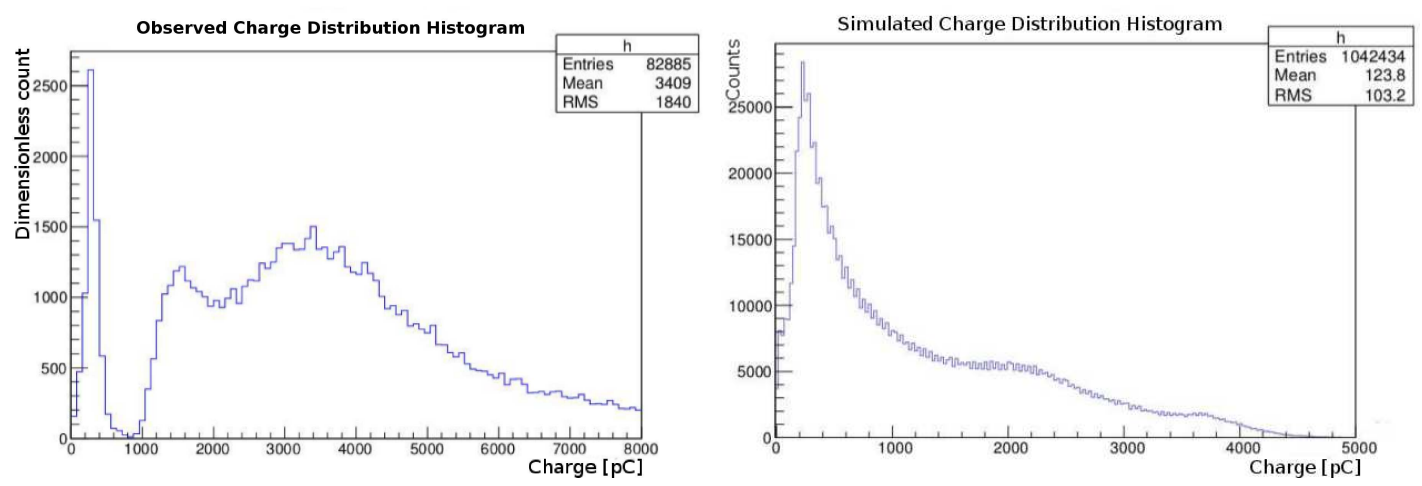

Figure 3: Left: Observed Charge Distribution Histogram. Right: Simulated Charge Distribution Histogram.

\subsubsection{Simulated Angular Distribution of particles}

To determine the particles that are lost due to the concrete coating, the Angular Charge Distribution Histogram is obtained. We proceed to simulate the flow of secondary particles from $0^{\circ}$ to $150^{\circ}$ in steps of $15^{\circ}$ for the azimuthal angle (Figure 4). In addition, the distribution of gammas, electrons, vertical muons (VEM) and lateral muons over the histogram is simulated (Figure 5).

\subsubsection{Energy loss}

Photons and electrons: The flux found in [8] shows that most of the electrons and photons that reach the detector do so with energies between 0.0001 and $1 \mathrm{GeV}$. When these particles travel through concrete are quickly absorbed or loose energy. Electrons and photons that manage to enter the detector will do so through the window mostly after noon.

Vertical muons: Vertical muons cross 3 floors that add $100 \mathrm{~cm}$ of concrete and around $10 \mathrm{~cm}$ of iron. A muon deposits $\sim 0.5 \mathrm{GeV} \equiv 500 \mathrm{MeV}$ in concrete and $\sim 0.15 \mathrm{GeV} \equiv 150 \mathrm{MeV}$ in iron. It has been considered that on average a muon deposits $5 \mathrm{MeV} / \mathrm{cm}$ in concrete and $1.5 \mathrm{MeV} / \mathrm{cm}$ in iron [13]. A VEM will loose $\sim 650 \mathrm{MeV}$ before reaching the detector.

Lateral muons: Mouns that pass through concrete and iron at different angles will go through different amounts of material. In concrete the maximum trajectory (at $45^{\circ}$ ), is around $1.4 \mathrm{~m}$, 

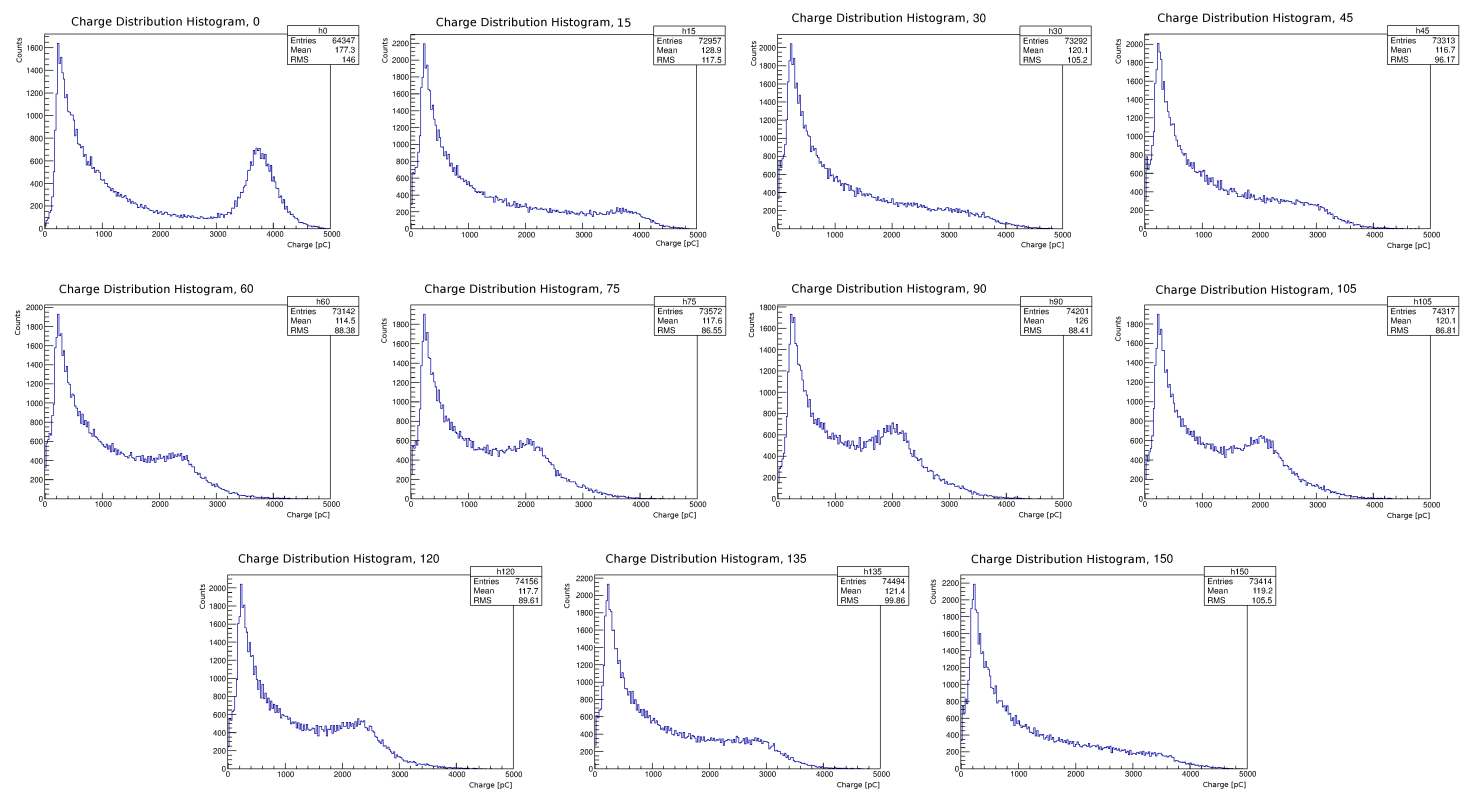

Figure 4: Simulated Angular Charge Distribution Histogram for the zenit angle between $0^{\circ}$ and $150^{\circ}$.

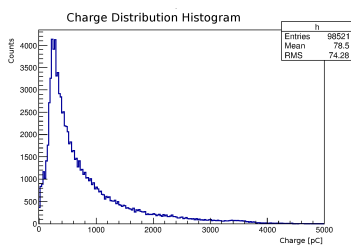

a

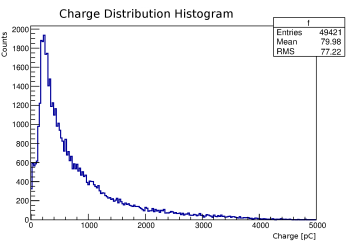

b

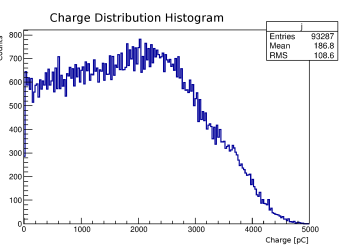

$\mathrm{c}$

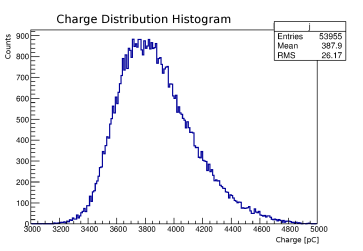

d

Figure 5: Simulated Charge Distribution Histogram for $(a) e^{-},(b)$ gammas, $(c)$ lateral muons and $(d)$ VEM.

so the maximum energy deposited is $707.1 \mathrm{MeV}$. For iron, the maximum trajectory is $14,1 \mathrm{~cm}$ with an energy deposited of $21,2 \mathrm{MeV}$. For lateral muons, the maximum total loss of energy is therefore $\sim 728,3 \mathrm{MeV}$ before reaching the detector.

\subsubsection{Vertical Equivalent Muon (VEM)}

The VEM is defined as: "The average charge deposited in a detector when it is completely crossed through by a single muon incident vertically on the center of its upper lid" ([6]) and it is obtained by adjusting the hump in the charge histogram.

Experimental VEM: The fit of a Gaussian curve around the $3500 \mathrm{pC}$ gives a VEM of 3350.983 $\pm 1163.45 \mathrm{pC}$ for the Politanque detector.

Simulated VEM: The simulated VEM is performed only with muons that enter perpendicular to the detector. The fit gives a VEM of $3799.8 \pm 249.46 \mathrm{pC}$.

The difference between this values can be explained by the energy loss explained in section 3.3.4. 


\section{Conclusion}

A WCD has been successfully installed in the facilities of the Escuela Politécnica Nacional. For the calibration we found: (1) the average width of a pulse is $154.8 \pm 22.1 \mathrm{~ns}$ (since it is close to the $160 \mathrm{~ns}$ that the data acquisition system registers, the correct acquisition of pulses by the system is verified), (2) the ideal working region for the PMT 5" is $1550 \pm 20 \mathrm{~V}$ with a threshold of $20 \mathrm{mV}$. The later data analysis gave away a dual behaviour of the global baseline, one at $-0.049 \pm 1.873 \times 10^{-5} \mathrm{mV}$ and another at $-0.041 \pm 0.0006 \mathrm{mV}$, therefore is recommended for future work that each output file has its own baseline. This tendency could be due to fluctuations of the main power supply.

It was found that $4.911 \pm 0.4455$ particles per unit of time go through the WCD. The detector has an effective area of $0.766 \mathrm{~m}^{2}$ and therefore the RATE is $6.4 \mathrm{~s}^{-1} \mathrm{~m}^{-2}$. The detection efficiency is $0.985 \%$ with respect to [15] and of the $1.067 \%$ with respect to the simulations carried out by [8].

On the other hand, the incidence of particles at different azimuth angles was simulated and the energy value deposited by muons, electrons and photons that traverse concrete and iron was calculated. It was found that the missing region in the observed charge histogram is due to the energy loss of electrons, photons and lateral muons that generates a reduction of the expected particle flux.

Using the background of particles that pass through the detector, it has been found that the experimental value of VEM is $3350.98 \pm 1163.4 \mathrm{pC}$ and simulated is $3799.8 \sim 249.4 \mathrm{pC}$. The registration capacity of the PMT with respect to the simulation is $88.2 \%$ due to the concrete and iron coating of the detector.

Finally, the culmination of this project is the one step forward for the flourishing of the LAGO collaboration within and outside the country. This ensures new projects oriented to the detection of cosmic rays using different geometry, coatings, electronics, PMT, among others. In addition, being the prototype tank of the Ecuadorian collaboration can be used as a learning engine for new generations.

\section{Acknowledgments}

The authors are grateful for the continuous support from the LAGO collaboration, Auger, CEDIA, Benemérita Universidad Autónoma de Puebla (BUAP) and the Escuela Politécnica Nacional.

\section{References}

[1] H. Asorey and S. Dasso for the LAGO Collaboration, LAGO: the Latin American Giant Observatory, in proceedings of 34rd International Cosmic Ray Conference, PoS(ICRC2015)247.

[2] LAGO collaboration, The Latin American Giant Observatory, http: / / lagopro ject . org/, cited 2016.

[3] J. Conde, Instrumentación para el Arreglo de Detectores de Superficie LAGO-México, Benemérita Universidad Autónoma De Puebla [PHD thesis, 2015].

[4] M. Suárez, Instalación de un Detector Cherenkov de agua para la detección de trazas de rayos cósmicos a 956 metros sobre el nivel del mar, Universidad Industrial de Santander [undergraduate thesis, 2011]. 
[5] A. Velarde, R. Ticona y P. Miranda, Proyecto Lago Bolivia, Revista Boliviana de Física, vol. 15(15), pp. 32-38.

[6] F. Yunior, Caracterización de Detectores Cherenkov en el Proyecto LAGO (Large Aperture GRB Observatory), Universidad de Los Andes [undergraduate thesis, 2016].

[7] I. Sidelnik for the LAGO Collaboration, The Sites of the Latin American Giant Observatory, in proceedings of 34rd International Cosmic Ray Conference, PoS(ICRC2015)665.

[8] C. Mantilla et al., Implementing a WCD detector system in Ecuador as part of the LAGO Project, in proceedings of SILAFAE edition X, Nuclear Physics B Proceedings Supplement, Vol 1.

[9] M. Audelo et al. for the LAGO Collaboration. Implementing a WCD detector system in Riobamba as part of the LAGO project. in proceedings of 33rd International Cosmic Ray Conference, ICRC2013.

[10] Pierre Auger Collaboration, The Pierre Auger Observatory Design Report, Second Edition, 14 of march of 1997 (2013 update).

[11] S. Vargas et al. for the Lago Collaboration. LAGO Ecuador, Implementing a set of WCD detectors for Space Weather research: first results and further developments, in proceedings of 34rd International Cosmic Ray Conference, PoS(ICRC2015)135.

[12] D. Allard et al., Use of Water Cherenkov Detectors to detect Gamma Ray Busrts at the Large Aperture GRB Observatory (LAGO), ELSEVIER Nuclear Instruments and Methods in Physics Research A, vol. 595, pp. 70-72.

[13] S. Vargas, Implementación y calibración de un detector terrestre Cherenkov de agua para rayos cósmicos en la Escuela Politécnica Nacional, Escuela Politécnica Nacional [undergraduate thesis, 2016].

[14] K. Romualdas, Photon Counting in Astrophotometry: Fundamentals and Some Advices for Beginners, Tr. J. of Physics, vol. 23, pp. 335-345.

[15] S. Vernetto, Detection of Gamma-Ray Bursts in the $1 \mathrm{GeV}-1 \mathrm{TeV}$ energy range by ground based experiments, Astropart.Phys., vol. 13, pp. 75-86. 